\section{Wingless: developmentally important genes that respond adversely to smoking}

\author{
Denise Al Alam, David Warburton
}

It has been observed by many authors that cigarette smoking results in long standing epithelial damage and interstitial inflammation within the lung. Quitting strongly reduces the risk of smoking associated lung diseases such as chronic obstructive pulmonary disease (COPD), but the underlying inflammation of the airways does not go away completely even after quitting smoking. So, a critical question for the pulmonary physician is: why is smoking cigarettes so bad for the airway lining and why are the inflammatory effects so long lasting?

Heijink et al, working with cultures of human primary bronchial epithelial cell (PBEC) biopsy specimens, now come with an intriguing new finding that cigarette smoking has significant adverse affects on certain members of the Wingless family, entraining a feed forward mechanism that increases epithelial cytokine production in response to cigarette smoke extract. ${ }^{1}$

The Wingless family comprises an important class of developmentally critical signalling ligands that conduct signals from the cell surface through the cytoplasm to the nucleus, where they activate coordinated sets of genes. These genes in turn regulate cell-cell communication in the embryonic through the adult stages of human and animal development and continue to be very important in airway epithelial differentiation as well as during airway epithelial healing (see Warburton et $a l^{2}$ for a recent review).

The colourful name Wingless was coined to describe the loss of function phenotype of fruit flies that lack this gene (they have no wings). Fruit fly geneticists, being somewhat perverse, always delight in giving active voice picaresque names to their loss of function phenotypes. Thus, conversely, signalling by Wingless ligands

Developmental Biology and Regenerative Medicine Program, Saban Research Institute, Children's Hospital Los Angeles, University of Southern California, Los Angeles, California 90027, USA

Correspondence to Dr David Warburton, Developmental Biology and Regenerative Medicine Program, Saban Research Institute, Children's Hospital Los Angeles, University of Southern California, Los Angeles, California 90027, USA; dwarburton@chla.usc.edu actually induces the correct differentiation of epithelial cells that form the wing structures in flies and this basic developmental function is conserved, but has been adapted during evolution all the way up to mice and humans, wherein Wingless genes play important roles in developmental branching of the airways. Because humans have undergone many gene reduplication events since they diverged from flies during evolution, there are several isoform copies of Wingless genes in the human genome, with multiple overlapping functions in tissue differentiation. These genes were also discovered independently at about the same time to be important in human breast cancer and therein were termed the Int family of genes. So, in the current gene nomenclature, Wingless plus Int was elided to Wingless (WNT).

The human WNT family of signalling ligands directs a complex network of downstream signalling events including what is termed a 'canonical' pathway as well as a 'non-canonical' pathway. In the canonical pathway WNT proteins bind to Frizzled (FZD) receptors and signal via second messengers to protect an important substance called $\beta$-catenin from proteolytic degradation; $\beta$-catenin is a powerfully pleiotropic transcriptional coregulator that sometimes enhances or sometimes represses transcription of many genes that promote tissue repair and regeneration such as growth factors, metalloproteinases and cytokines. Meanwhile, the non-canonical branch of WNT signalling is $\beta$-catenin independent and can induce signalling pathways involved in regulating cell survival, immune responses and cytoskeletal proteins such as p38, calcium ion concentrations and RhoA. Several WNT pathway regulators have been reported, including Dikkopf proteins, WISE, secreted FZD receptor proteins, the Wnt inhibitory factor (WIF) and the R-spondin family. ${ }^{3}$ Canonical Wnt ligands comprise Wnt1, Wnt2, Wnt3a and Wnt10b, while other Wnt ligands such as Wnt5a and Wnt11 only mediate $\beta$-catenin-independent transcriptional activation.

During lung development, Wnt signalling determines cell proliferation, differentiation and lineage commitment. Several Wnt proteins and receptors are expressed in the mouse embryonic lung at different developmental stages. In addition, using three different Wnt reporters, we have shown that Wnt signalling is active in both lung epithelium and mesenchyme as well as in airway smooth muscle cells. ${ }^{4}$ Therefore, one would expect that any alteration of Wnt signalling would affect the development of at least one of these compartments of the lung. Interestingly, both inhibition and excessive activation of Wnt signalling lead to abnormal lung development in mice. Recent studies reported the presence and the spatio-temporal expression of several WNT ligands (WNT7b, WNT2), receptors (FZD4, FZD7), regulators and signal transducers (B-CATENIN, AXIN2, $A P C)$ in human embryonic lung, ${ }^{5}$ but functional studies in the developing human lung are missing thus far.

Furthermore, we showed that Wnt signalling is strongly activated following naphthalene injury of bronchial epithelium and during injury repair in mice. ${ }^{4}$ However, recently, more light was shed on the role of this developmentally important pathway in human lung disease. Excessive WNT signalling has been implicated in pulmonary fibrosis as well as in some types of lung cancer, including smoke-induced lung cancer (see review by Konigshoff and Eickelberg). ${ }^{3}$ Moreover, genome-wide association studies have revealed a clear association between WNT pathway genes and asthma in children. ${ }^{6}$ Similar studies in idiopathic pulmonary arterial hypertension showed differential expression of components of both the canonical and noncanonical WNT pathways in patient's vessels, versus transplant donor vessels, microdissected by laser capture. ${ }^{7}$ Thus, WNT signalling seems to be increasingly identified as a potential target in certain intractable forms of lung disease.

Studies in mice exposed to smoke from 3 weeks before conception until delivery showed that maternal smoke exposure strikingly decreased Wnt signalling pathway genes ( $\beta$-catenin, Fzd7) as well as Foxa2 in the lungs of the neonatal offspring, ${ }^{8}$ thus leading to impaired alveolarisation and abnormal lung development. Moreover, both a decrease of the Wnt target genes $\beta$-catenin and TCF7L1 and an increase of the Wnt inhibitor SFRP2 were reported in bronchial epithelial cells of both 'healthy' and COPD smokers.' In addition, decreased expression of canonical Wnt signalling targets was observed in experimental mouse models of emphysema induced by elastase instillation or by smoke exposure. Taken together, these studies suggest therefore that canonical Wnt pathway signalling may exert beneficial as well as 
potentially adverse effects in COPD and emphysema. 910

Interestingly, it has been shown that Wnt5a, a non-canonical Wnt, induces upregulation of pro-inflammatory cytokines (interleukin (IL)-6, IL-8 and IL-15) in rheumatoid arthritis, ${ }^{11}$ while WNT5a expression is also increased in psoriasis, another chronic inflammatory disorder. ${ }^{12}$ WNT5a binding to FZD5 also potentiates microbially-induced inflammation in macrophages and enhances the release of IL-12. ${ }^{13}$ Non-canonical Wnt signalling also leads to calcium ion release and activation of protein kinase $\mathrm{C}$ and CaMKII, which can in turn activate $\mathrm{NFkB}, \mathrm{AP} 1$ and nuclear factor of activated $\mathrm{T}$ lymphocytes (NFAT) pathways. The NFkB family of transcription factors is one of the common pathways activating the release of pro-inflammatory cytokines and chemokines in different forms of inflammation.

Inflammation and tissue damage are hallmarks of COPD. Now, Heijink et al specifically show that expression of the WNT-4 ligand isoform is significantly increased in PBECs from COPD patients, versus 'well' smokers and non-smokers. Moreover, addition of WNT-4 to PBEC cultures strongly potentiates cigarette smoke extract-mediated induction of a potent suite of pro-inflammatory cytokines including IL-8, IL-6, CCL-5 and CCL2 as well as the vascular endothelial growth factor. ${ }^{1}$ WNT-4 is thought to act through both the canonical and noncanonical Wnt pathways. But in this study, the authors did not see any evidence of change in $\beta$-catenin activity, while an increase in phosphorylated p38 was observed, thus suggesting that, in COPD, WNT4 acts preferentially through the non-canonical Wnt pathway to induce the release of inflammatory mediators. However, it will be interesting to investigate whether WNT4 also activates the NFkB pro-inflammatory pathway.

Although there is evidence in the literature of cross talk between the canonical and non-canonical Wnt signalling pathways, these two pathways seem to play opposing or balancing roles in COPD, as well as following smoke exposure. It is now quite well accepted that targeting the Wnt pathway could lead to the development of therapies for several diseases, yet the question remains which branch of the Wnt pathway and where and how to target it?

Tobacco smoking is associated with around $85 \%$ of all human cases of COPD. The novel finding in the accompanying paper is that cigarette smoke seems to have induced WNT-4 signalling in the lung epithelium of COPD patients, which in turn, by activating WNT downstream signalling, potentiates the effects of smoke extract on inflammatory cytokine production by PBECs, thus driving a potentially very nasty feed forward, self-reinforcing loop that could explain why smoking leads to persistent airway inflammation, particularly in COPD. Or to paraphrase the instructions on British fireworks: light the blue touch paper, inhale and stand clear! Yet another reason not to start smoking, or if you have started, quit as soon as possible, before the WNT-4 feed forward loop on cytokine production in the lung epithelium can become irreversibly established, supporting chronic airway inflammation as in COPD, a circumstance that could 'grow you a premature set of wings'.

Funding DW acknowledges the support of Pasadena Guild, Children's Hospital Los Angeles, Webb Foundation, Garland Foundation, California Institute for Regenerative Medicine (CIRM TG2-01168, RN2-00946, CL1-00507-1), NIH/NIGMS (1R01GM096195), NIH/ NHLBI (1RO1HL104258-01) and NIH/NIEHS (1D43ES02286201).

\section{Competing interests None.}

Provenance and peer review Commissioned; internally peer reviewed.

To cite Al Alam D, Warburton D. Thorax 2013;68:703-704.

Published Online First 23 April 2013

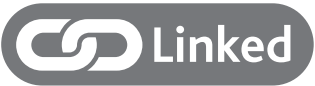

http://dx.doi.org/10.1136/thoraxjnl-2012-201667

Thorax 2013;68:703-704.

doi:10.1136/thoraxjnl-2013-203249

\section{REFERENCES}

1 Heijink $I H$, de Bruin $H G$, van den Berge $M$, et al. Role of aberrant WNT signalling in the airway epithelial response to cigarette smoke in chronic obstructive pulmonary disease. Thorax 2013;68:709-16.

2 Warburton D, El-Hashash A, Carraro G, et al. Lung organogenesis. Curr Top Dev Biol 2011;90:73-158.

3 Konigshoff M, Eickelberg 0. WNT signaling in lung disease: a failure or a regeneration signal? Am J Respir Cell Mol Biol 2010;42:21-31.

4 Al Alam D, Green M, Irani R Tabatabai, et al. Contrasting expression of canonical Wnt signaling reporters TOPGAL, BATGAL and Axin2(LacZ) during murine lung development and repair. PLoS One 2011;6:e23139.

5 Zhang M, Shi J, Huang $Y$, et al. Expression of canonical WNT/beta-CATENIN signaling components in the developing human lung. BMC Dev Biol 2012;12:21

6 Sharma S, Tantisira K, Carey V, et al. A role for Wnt signaling genes in the pathogenesis of impaired lung function in asthma. Am J Respir Crit Care Med 2010:181:328-36.

7 Laumanns IP, Fink L, Wilhelm J, et al. The noncanonical WNT pathway is operative in idiopathic pulmonary arterial hypertension. Am J Respir Cell Mol Biol 2009;40:683-91.

8 Blacquiere MJ, Timens W, van den Berg A, et al. Maternal smoking during pregnancy decreases Wnt signalling in neonatal mice. Thorax 2010;65:553-4.

9 Wang R, Ahmed J, Wang G, et al. Down-regulation of the canonical Wnt beta-catenin pathway in the airway epithelium of healthy smokers and smokers with COPD. PLOS One 2011;6:e14793.

10 Kneidinger $\mathrm{N}$, Yildirim AO, Callegari J, et al. Activation of the WNT/beta-catenin pathway attenuates experimental emphysema. Am I Respir Crit Care Med 2011;183:723-33.

11 Sen $\mathrm{M}$, Lauterbach K, El-Gabalawy $\mathrm{H}$, et al. Expression and function of wingless and frizzled homologs in rheumatoid arthritis. Proc Natl Acad Sci USA 2000;97:2791-6.

12 Reischl J, Schwenke S, Beekman JM, et al. Increased expression of Wnt5a in psoriatic plaques. I Invest Dermatol 2007;127:163-9.

13 Blumenthal A, Ehlers S, Lauber J, et al. The Wingless homolog WNT5A and its receptor Frizzled-5 regulate inflammatory responses of human mononuclear cells induced by microbial stimulation. Blood 2006;108:965-73. 